\title{
A description of the new technologies used in transforming dental education
}

IN BRIEF
- This paper is the first in a series of 16
which reviews the use of information
and communication technology (ICT)
in dentistry.
- It considers how new technologies are
transforming dental education.
It concludes that the development of
appropriate technology should be driven
by educational needs, not the search for a
purpose for the tools themselves.

\author{
L. Feeney, ${ }^{1}$ P. A. Reynolds, ${ }^{2}$ K. A. Eaton ${ }^{3}$ and J. Harper ${ }^{4}$
}

Information and communications technology (ICT) is a universal term describing the use of technology and media to disseminate and share knowledge and information. Educational media provide a means by which information can be communicated between teacher and learner. In this context, the aim of this digest is to summarise and describe the spectrum of tools available. All technologies used in education have their strengths and weaknesses. Variety needs to be balanced by economy. However, it must be remembered that technology is not the overriding issue. The design of the learning environment and the learning experience itself are far more important. Technologies are generally flexible and can be used in a variety of ways. Knowledge of these technologies will help guide appropriate selection. The following tools are described: 1. Telecommunications services; 2. Communications tools (social software) including email, newsgroups, weblogs and wikis; 3. Richmedia in interactive training and learning; 4. Compact discs (CDs) and digital video discs (DVDs); 5 . Virtual learning environments (VLE); 6. Sophisticated communications - videoconferencing, webcasting and podcasts; 7. Interactive television; and 8. Virtual reality.

\section{E-LEARNING IN DENTISTRY}

Section A: Teaching and technology

1. A description of the new technologies used in transforming dental education

2. Seeing is believing: dental education benefits from developments in videoconferencing

3. Webcasting: casting the web more widely

4. Top of the pops - CD-ROM and DVDs in dental education

Section B: Informatics: better informed

by systems and services

5. Better informed: an overview of health informatics

6. Better informed in clinical practice a brief overview of dental informatics

7. Digital clinical records and practice administration in primary dental care

Section C: Impact of e-learning in

dental education

8. Remember the days in the old school yard: from lectures to online learning

9. An intricate web - designing and authoring a web-based course

10. The many faces of interaction

11. Supporting the learner and teacher online

12. Making a mark - taking assessment to technology

13. Continuing professional development and ICT: target practice

14. Assuring quality

Section D: A connected future

15. Nine years of DentEd: a global perspective

16. A vision of dental education in the third millenium
This overview of the information and communications technology (ICT) tools available for the education and training of dentists and their team is the first in a series of articles revealing how the practice of teaching and learning is influencing technology in dental training. In a rapidly changing world this digest is a snapshot of the technology at the time of preparation; developments will march on apace. Good pedagogical practices will inevitably continue to flex and challenge technological advances.

e-Learning has been defined as 'learning in a way that uses information and communication technologies.'

\footnotetext{
'Knowledge, Information \& Quality Consultant, Royal College of Surgeons of Ireland's International School of Healthcare Management, 123 St. Stephens Green, Dublin 2, Ireland; ${ }^{2 *}$ Professor of Dental Education, Centre of Flexible Learning in Dentistry, King's College London Dental Institute, Floor 3, Strand Bridge House, 138-142 Strand, London, WC2 $1 \mathrm{HH} ;{ }^{3}$ Visiting Professor, UCL Eastman Dental Institute, 256 Gray's Inn Road, London, WC1X 8LD; ${ }^{4}$ T Manager, The Dental Institute at Guys King's and St Thomas' NHS Hospitals, King's College Hospital, Denmark Hill, London, SE5 9RW ${ }^{*}$ Correspondence to: Professor P. A. Reynolds Email:P.A.Reynolds@kcl.ac.uk
}

\section{Refereed Paper}

DOI: 10.1038/bdj.2007.1185

${ }^{\circ}$ British Dental Journal 2008; 204: 19-28
ICT makes possible the interactive sharing of knowledge and information by providing ever newer and faster ways of delivering and accessing information, innovative methods of 'real-time' communication and new ways to carry out all types of business. 'Real-time' communication is used to describe a number of different computer features, for example real-time operating systems are systems that respond to input immediately. ICT is ensuring that more and more information is available in the public domain. This can lead to improved efficiency, transparency and accountability but can also be overwhelming. The 'transformational' potential of ICT may be immense but it is imperative that the dental profession take advantage of the 'knowledge-based' environment and apply the technology to reap both social and economic rewards in the future. ${ }^{2}$

A major indicator of the success of any technological development is the point when it ceases to be treated as novel or new and becomes the norm. Examples in ICT include email, Internet connectivity, CD-ROM access (compact discread only memory) and DVDs (digital versatile/video disc). The establishment 
of industry-wide reliable standards, decreasing costs, the availability of broadband or ADSL (asynchronous digital subscribers line) and Internet protocol (IP) as well as increasingly robust networks are creating an environment where the use of ICT and ICT tools is now accepted. However, the pioneers of the technology did not expect it to entirely replace person-to-person interaction or communication. ${ }^{3}$ Indeed, a whole pedagogy has evolved over the past few decades that has driven and supported the appropriate and effective use of technology and the media in learning and teaching. ${ }^{4,5}$

\section{TELECOMMUNICATIONS SERVICES}

Telecommunications technologies and services are platforms or 'vehicles' which allow all types of data or information transmission, from voice to video. Advances in these technologies and services are providing a wider range of possibilities that can have a positive impact upon the 'electronic' or 'e-learning' environment. Essentially these new services improve the delivery and accessibility of knowledge, information and expertise together with educational courses in general.

Two developments in particular 'broadband' and 'wireless' - are potentially having the greatest influence.

Broadband has been the cornerstone of the UK Government's ICT policy, which by 2005 had aimed to create the most extensive and competitive broadband market and to exploit its full potential in education. According to the Broadband Stakeholder Group (BSG) at that time, 'Broadband can transform the learning experience for students as it can expose them to a range of exciting and innovative learning content that was previously either inaccessible or impractical in the narrowband environment'. Its greater bandwidth (the speed of data or information transferred in a fixed time) over the dial-up (standard telephone line) alternative allows for the faster transmission of larger files of data in multimedia form, while its 'always-on' nature not only reduces user costs, but also allows for increased interaction. ${ }^{6}$
In the UK broadband is commonly delivered using asynchronous digital service lines (ADSL) - a transmission technology that allows more data to be sent over existing copper telephone lines. This supports speeds of 1.5 to $24 \mathrm{Mbps}$ with ADSL 2+ (megabits per second - a convenient measure of the data rate in digital communications) when receiving data (known as the downstream rate) and from 16 to $640 \mathrm{Kbps}$ and above when sending data (known as the upstream rate). This availability and decreasing prices is boosting the use of broadband. However, ADSL is not ideal for every educational application, most notably videoconferencing, due to this dual speed characteristic. Symmetric services (SDSL), offering higher speeds in both directions, are now available from BT and others and these 'bandwidth-on-demand' services allow users to deploy higher bandwidths when required by demanding applications such as videoconferencing.

More problematic for ASDL users is the lack of consistent response inherent in the technology and the variability of speed depending on the number of subscribers in any one dedicated link. Given the ADSL speed and latency (the time taken for the signal to travel from source to destination) it is sometimes not fast enough for real-time applications and is also subject to 'packet loss' (a 'packet' is a piece of a message sent over a network such as the Internet). Internet protocol (IP) networks (an IP network such as the Internet is something like the postal system for your data or information, allowing your computer to address a packet and drop it onto a network safe in the knowledge it will reach its destination) are managed dynamically and if there is a problem, packets can be discarded and re-requested. However, the interruption during real-time uses such as videoconferencing can lead to the irreplaceable loss of parts of the sound and vision and, with it, the lack of true interaction. This problem is being addressed by a process called conditioning that reduces the latency and contention problems.

Educators are not totally unfamiliar with broadband. The JANET and SUPERJanet networks provide what is virtually an 'on-campus' environment for education establishments. Such networks or 'intranets' as they are more commonly known, are built to higher standards than the public Internet with better security, stability and faster speeds, and, therefore are far less likely to suffer from latency and packet loss difficulties. However, as the BSG report points out, 'The full educational impact of broadband can only be realised when off-campus connectivity (ie learners in the home) mirrors that on-campus. ${ }^{6}$

Outside the UK, similar developments in other countries are also increasing broadband's deployment and uptake. All the EU15 (the old members prior to 1 May 2004) Member States have national broadband strategies and the new member states have also tabled theirs.

The December 2005 statistics from the Organisation for Economic Co-operation and Development (OECD) show that there were 158 million broadband subscribers in OECD countries. The largest number, not surprisingly, were in the US with over 49 million; the UK had over 9.5 million while in terms of penetration, the list was headed by Iceland, Korea, the Netherlands and Denmark with more than 25 subscribers per 100 inhabitants. $^{7}$

It is not surprising that the developed world is ahead in the broadband stakes. Developing countries with their poorer fixed line telecommunications infrastructure are not in such a happy position. However, they may be able to catch up through broadband wireless technology which is easier, quicker and cheaper to deploy.

For many, wireless means GSM (global system for mobile communications) mobile phones operating at 9.6 Kbits and primarily used for voice and texting. However, general packet radio service (GPRS), a derivative of GSM and using IP, provides a data service theoretically capable of speeds up to 172.2 kilobits per second (Kbps), although limitations mean actual speeds are a lot lower. With a typical throughput of $40 \mathrm{Kbps}$, email and Internet access on the move are perfectly feasible.

Further speed enhancements are being promised by EDGE (enhanced data rates for GSM evolution). This offers up to three times the data capacity of GPRS and allows the delivery of advanced 


Table 1 Data speed abbreviations and their meanings
\begin{tabular}{l|l|l|l}
\hline Abbreviation & Spoken & Bits per second & Alternative abbreviations \\
\hline Kbps & Kilobits & 1,000 & Kb or Kbits \\
\hline Mbps & Megabits & $1,000,000$ & Mb or Mbits \\
\hline Gbps & Gigabits & $1,000,000,000$ & Gb or Gbits
\end{tabular}

\begin{tabular}{|c|c|c|c|}
\hline Account type & Speed & Bits per second & Time to download $1 \mathrm{MB}$ \\
\hline Dial-up & 56 Kbps & 56,000 & 143 seconds \\
\hline ISDN & $128 \mathrm{Kbps}$ & 128,000 & 63 seconds \\
\hline Broadband & $1 \mathrm{Mbps}$ & $1,000,000$ & 8 seconds \\
\hline
\end{tabular}

mobile services such as downloading of music and video clips and full multimedia messaging as well as Internet access and email on the move.

Third generation (3G) networks have higher speeds more suited to educational applications. Speeds of 2 Megabits per second (Mbps) are achievable although, again, users will probably experience lower speeds of, say, $144 \mathrm{Kbps}$. However, even at these speeds, new applications such as web-browsing and file transfer are possible. Already $3 \mathrm{G}$ is being upgraded: HSPDA (high speed downlink packet access) is being deployed to increase the speed from network to user by a factor of 4-5, making it suitable for mobile broadband multimedia services including mobile TV.

The constraint for such applications would seem to be the screen of a typical mobile phone. However, 3G services are also suitable for use with other terminals: 3G data cards are available for slotting into laptops, personal digital assistants (PDAs) and notebooks, turning them into full-scale $3 \mathrm{G}$ terminals.

However, for such terminals $3 \mathrm{G}$ does not offer the sole method of connectivity. Operating at $11 \mathrm{Mbps}$ or $54 \mathrm{Mbps}$ and higher (depending on the standard utilised), wireless local area networks (Wi-LANs) have both on-campus and off-campus roles. In the former they are ideal for extending a network infrastructure to additional buildings or to buildings that are difficult to connect via cable. Off-campus their usefulness is for students (and tutors) who wish to access course material and information when they are away from the campus. This possibility has been facilitated by the launch of public Wi-LAN services that are accessible via 'hot-spots' installed in a variety of locations including coffee shops, hotels, airports and railway stations.

To complete the Wi-family, WiMax is a method for broadband access providing high throughput broadband connections over long distances; it is the wireless equivalent of the 'last-mile' cable connection into the home, office or college.

One other wireless technology warrants a brief mention: Bluetooth. This is named after a 10th century Danish king who unified Christianity in Denmark and whose name conjures up an interesting differential diagnosis. In technical terms, however, Bluetooth is a shortrange radio technology that connects different pieces of electronic equipment being used for cable-less short-distance links, eg between a PC and a printer. It is also the preferred medium for the connection between headsets and mobile phones and may well have future wireless applications at the dental chair side.

\section{Note - the speed of data or information}

The letters bps are short for bits per second, a measure of speed. Simply stated, the higher the bps, the less time it takes for data or information to transmit and thus it is similar to miles per hour (mph) in that sense. As a rule, bps is used as a measure of the speed of getting data from one computer to another across a network (including the Internet). ' $\mathrm{K}$ ' stands for a thousand, ' $M$ ' for a million and ' $G$ ' for a billion. Table 1 sums it up.

To demonstrate how the speeds relate to one another in terms of 'waiting time', the Table 2 compares the amount of time it would take to download (or copy) a $1 \mathrm{MB}$ (mega byte) file from a computer on the Internet to your own computer (a byte is equal to 8 bits, and thus a $1 \mathrm{MB}$ file is roughly 8,000,000 bits).

\section{COMMUNICATION TOOLS}

ICT is making the interactive sharing of knowledge and information possible through increasingly faster ways of communicating and accessing information. Email, newsgroups, mailing lists and chat rooms are well-known forms of communication media. These may be described as follows:

- Email (electronic mail) is a messaging service that allows text messages and attached files to be sent and received to and from a known user address using a computer network (computers linked together). This may be locally within a company or institute LAN (local area network) or a WAN (wider area network) such as the Internet; while it is not $100 \%$ secure, it is relatively confidential. In January 2007, 1.1 billion connections were estimated worldwide with 37 million in the UK (ranking sixth in the world behind USA, China, Japan, Germany and India) and 210 million in the USA. ${ }^{8}$ An IDC (International Data Corporate) study entitled E-mail usage forecast and analysis, 2000-2005 suggests that the number of emails sent on an average day reached 10 billion worldwide in 2000 and, by 2005, this was expected to triple to a staggering 35 billion emails sent daily ${ }^{9}$

- Newsgroups (or forums) are 'online' discussion groups which allow textbased messages to be openly accessible at a public site on the Internet. Messages may come from anywhere with no verification of the identity of the sender and can be read by anyone. Each newsgroup is generally concerned with one particular subject or a common interest of a group of 


\begin{tabular}{|c|c|c|}
\hline & Advantages & Disadvantages \\
\hline Accessibility & $\begin{array}{l}\text { Individuals and groups have instant } \\
\text { access to information, which keeps them } \\
\text { constantly up to date. Able to address large } \\
\text { numbers. }\end{array}$ & $\begin{array}{l}\text { Time consuming to answer all the } \\
\text { emails. Instant responses may be } \\
\text { sent which may be regretted later. }\end{array}$ \\
\hline $\begin{array}{l}\text { Collaborative } \\
\text { learning }\end{array}$ & $\begin{array}{l}\text { Student or research group can be easily } \\
\text { contacted by the teacher for the provi- } \\
\text { sion of answers, setting of tasks or group } \\
\text { discussions. The teacher or trainer remains } \\
\text { informed of ongoing discussions and com- } \\
\text { munications. }\end{array}$ & $\begin{array}{l}\text { Too transparent, students may feel } \\
\text { they have little privacy from their } \\
\text { peers or teachers }\end{array}$ \\
\hline Resources & $\begin{array}{l}\text { Provides students with rapid access to } \\
\text { frequently changing local and worldwide } \\
\text { opinions and information throughout their } \\
\text { training programmes. Provides a variety of } \\
\text { resources for evidence-based work. }\end{array}$ & $\begin{array}{l}\text { Information overload which can } \\
\text { also generate more paper. Little } \\
\text { quality control of many web-based } \\
\text { resources. }\end{array}$ \\
\hline Administration & $\begin{array}{l}\text { Provides teachers with an excellent and } \\
\text { efficient administrative information tool. } \\
\text { Fosters tutoring with students as or when } \\
\text { required. }\end{array}$ & $\begin{array}{l}\text { Need to be constantly in touch so } \\
\text { as not to miss changes to arrange- } \\
\text { ments. Students also like face to } \\
\text { face meetings }\end{array}$ \\
\hline ICT training & $\begin{array}{l}\text { Users become familiar and confident with } \\
\text { the use of this technology. It can also can } \\
\text { stimulate wider interest in ICT }\end{array}$ & $\begin{array}{l}\text { Some may find the use and power of } \\
\text { technology daunting }\end{array}$ \\
\hline
\end{tabular}

people with different sub-discussions automatically attached. These discussions are usually listed in 'threads' to allow ease of access and continuity

- Mailing lists (or distribution lists) are a hybrid of email and newsgroups. Participation requires a user to 'sign-in' and allows members to send a message by email to the address of the mailing list, which redistributes it automatically to all the other participants. The discussions' threads are not grouped as in the newsgroup but by using email; the name and address of the sender is usually identifiable

- Chat rooms are online areas allowing a synchronous exchange of written messages between several people. Each time a participant types and sends a message, the dialogue is immediately visible to all in the chat room. Chat rooms may be set up on a local network, but are more commonly found on the Internet. The chat rooms can be public, in which case participants may be anonymous and use pseudonyms. They can also be private within a local learning or communications environment.

Table 3 summarises the educational advantages and disadvantages of email, newsgroups, mailing lists and chat rooms.

With the advent of Web 2.0, more users are connecting and collaborating democratically through social software such as Facebook ${ }^{\mathrm{TM}}$ (www.facebook.com), MySpace ${ }^{\mathrm{TM}}$ (www.myspace. com) and $\mathrm{Bebo}^{\mathrm{TM}}$ (www.bebo.com). Both informal and formal learning can occur when these tools are used. Social bookmarking software such as del. icio.us (http://del.icio.us) and CiteULike (www.citeulike.org) enable lists of bookmarked sites to be made available to others using a search tool. Multimedia sharing software of images, videos and podcasts is made possible through websites such as Flickr ${ }^{\mathrm{TM}}$ (www.flickr. com), YouTube (www.youtube.com) and Odeo (http://odeo.com), respectively. ${ }^{10}$ Blogging and wikis are discussed below and RSS feeds (real simple syndication) are discussed in Section 6. RSS feeds are a method of collating and distributing updates and news from designated websites. ${ }^{10}$

- Blogging (weblogging): a more recent use of online communication is the 'blog'. This is a personal online journal accessible by all web users. Its contents can vary from anything from an individual's personal diary to an academic exercise. Blogs can be a useful collaborative aid, allowing students to air their views and opinions on their courses and coursework, and access those of their contemporaries ${ }^{11}$

- Wikis are collaborative web applications that allow users to edit content. Wiki is short for wiki wikiweb, where 'wiki wiki' means quick in Hawaiian. ${ }^{11}$ An example of current use is updating the dental therapeutics knowledge base as an online task for undergraduate dental students. ${ }^{12}$

\section{RICH MEDIA IN INTERACTIVE TRAINING AND LEARNING}

The cornerstone of interactive training and learning is the effective use of rich media The term 'rich media' is synonymous with interactive multimedia, which has been defined as the combination of sight, sound and the senses through the use of contemporary technology. ${ }^{13}$ Indeed, a new grammar is developing that incorporates new meanings based on semiotic theory that are evolving from the use of such sense-enhancing tools. ${ }^{14}$ Multimedia interactive learning can combine text and illustrations, videos, sound and animation with feedback. This may include student questions, testing and tracking of the students' progress. Interactive learning has several additional benefits including:

- The delivery of consistent standardised training across an organisation, school or university at a lower cost than using 'live' instructors, yet still ensuring students receive the same level of instruction. ${ }^{3}$ This delivery allows the student to study at the most convenient times both during and outside normal work hours with reduction in timetabling constraints

- Electronic records of the presented material and of the responses to tasks set for the students can enable and encourage learning

- Continuing education is an excellent model for the use of interactive training in conjunction with online testing and verification that the course has been completed satisfactorily.

Interactive training can be delivered on a stand-alone or networked environment. Both delivery methods provide 
similar benefits, including:

- Ease of accessibility and cost: interactive training allows personnel at various locations, or those working shifts, equal opportunity to learn. Although initial development or purchase of course materials and hardware requires a significant investment, live training can be much more costly ${ }^{3}$

- Delivery: learning is interactive, personalised, flexible and self-paced, as opposed to instructor-led classroom training that is sometimes didactic and rigid. Performance results can also be fed back to the student and the system adapted to the learning needs of the student

- Educators: it allows a good educator to serve or facilitate many students, and permits access to geographically remote experts

- Networked interactive learning also has additional specific benefits including ease of content updating, monitoring student data and group activities ranging from text-based discussion groups to live audio and video.

\section{COMPACT DISCS AND DIGITAL VIDEO DISCS}

\section{Compact discs}

Compact discs (CDs) are capable of storing digital information. The two most popular types of CDs are those used by the music industry to store digital recordings and CD-ROMs (compact disc read-only memory) used to store computer data and programs. The CD-ROM is a type of optical disc (an optical disc is a storage medium using laser technology to read and write data) capable of recording large amounts of data up to 650 megabytes (a megabyte is a unit of computer storage approximately equivalent to 1 million characters), which is about 300,000 text pages.

The CD-ROM was originally a 'readonly' disc that cannot be erased or have new data added once it is manufactured or produced, however read-write discs are now commonplace. All CD-ROMs conform to a standard size and format, thus allowing any type of CD-ROM to be accessed in any CD-ROM drive. A

\begin{tabular}{|c|c|}
\hline & DVD format application \\
\hline DVD-video & Interactive or linear video programs \\
\hline DVD-ROM & Multimedia programs or data storage of any type, requires a computer \\
\hline DVD-R* & Record-once format used in developing DVD-video or DVD-ROM programs \\
\hline DVD-RAM** & Re-recordable format, used as a computer peripheral to store files \\
\hline DVD-audio & Audio only format, specifications still in development \\
\hline
\end{tabular}

CD-ROM drive is a component of a computer that can read information from a CD-ROM. In addition, computer CD-ROM drives are capable of playing audio CDs as they share similar technology.

CD-ROMs are particularly well-suited to information that requires large storage capacity, for example pictures or video. They have been used as a vehicle to provide a wide range of computer-aided learning programmes for the continuing professional education of dentists in England. ${ }^{15}$ The arrival of compact recordable discs (CD-Rs) and compact re-writable discs (CD-RWs) has expanded the versatility and uses of the $\mathrm{CD}$.

\section{Digital versatile discs (DVDs)}

DVD is the abbreviation for either digital versatile disc or digital video disc - both terms are interchangeable. DVD technology was launched in 1995 as a major step forward in optical disc technology, since when it has become the most successful consumer electronics product of all time. ${ }^{16}$ It can address technology needs that neither the earlier 'laser disc' nor the CD-ROM alone can fully meet. DVD movie discs and home entertainment DVD players are now readily available but there are also applications for teaching and learning.

In effect, DVD is a second generation CD-ROM combining the best features of laser disc and CD-ROM programs, creating a multimedia platform that can deliver high quality, full motion video and CD quality audio. It also provides an on-screen interactive navigation capability with branching (branching allows the user to select a particular lesson from a list of menu options or to quickly navigate through the program content).
With these features, educators can use either a DVD-video player or DVD-ROM computer system as tools for providing group or individual instruction. ${ }^{16} \mathrm{~A}$ DVD is often compared to a compact disc as it is looks and feels the same. However a DVD can hold two sides of information on the same disc, and each side can contain two layers of data.

\section{DVD formats}

According to the DVD Forum (an international association of hardware manufacturers, software firms and other users of DVDs that promotes the broad acceptance of DVD products worldwide), there are five specified DVD disc formats (Table 4).

The two most important DVD formats for education are DVD-video and DVDROM as these formats can be used to deliver educational content to schools, universities and libraries. The format of the disc describes how information is stored on the disc and which type of hardware system must be used to play the disc.

DVD-video offers additional features for teachers based on the educational objectives of the program. It can be a highly interactive platform that does not require a computer and is easy to use by both teachers and students.

High Definition (HD) or Blu-Ray (BD) optical disks are also available and can store up to $60 \mathrm{~GB}$ of videos with line resolutions almost double $(1,080)$ that of normal PAL (phase alternating line) TVs (576). Latterly, the use of USB (universal serial bus) hard drives (USB sticks) has allowed fast and reliable storage and transfer of large amounts of data (many gigabytes), which is already numbering the days of the discs. 


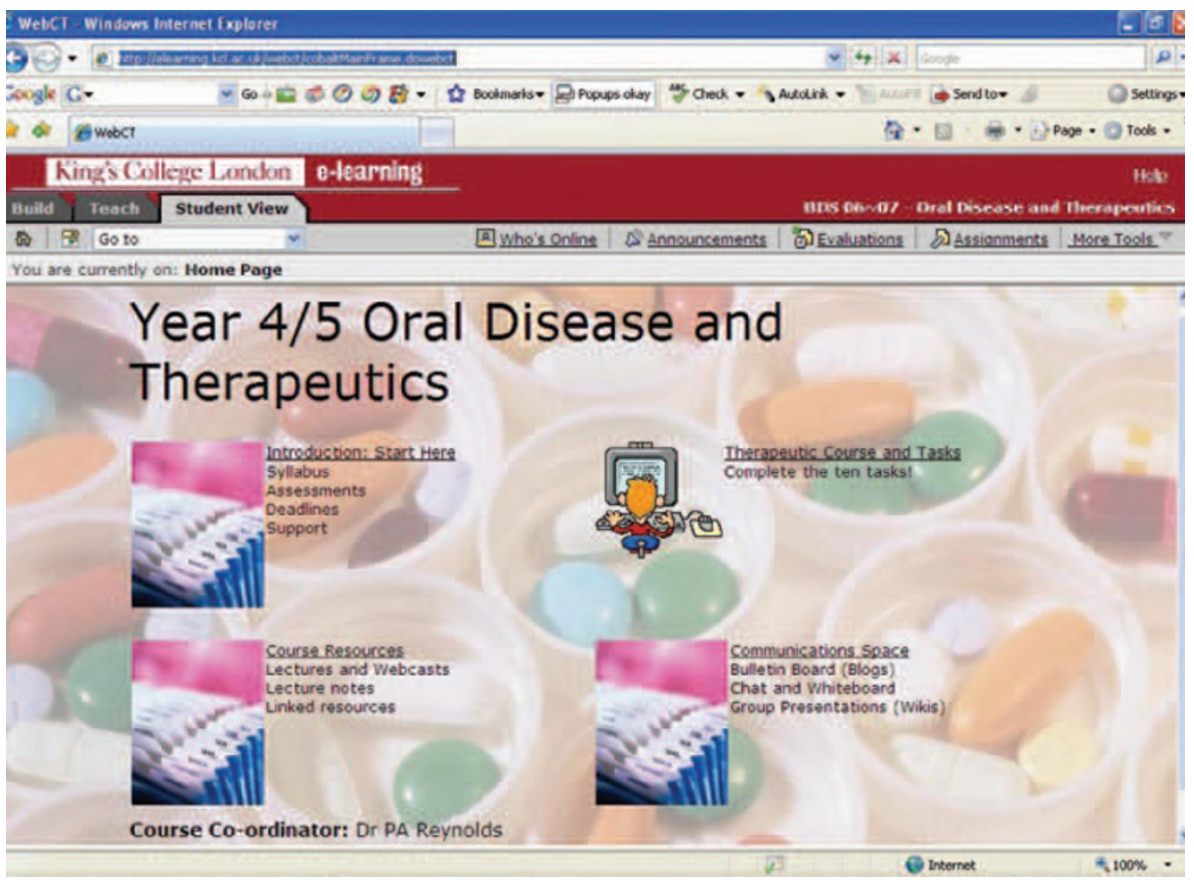

Fig. 1 Screenshot of homepage of Year 5 dental undergraduate web-based course

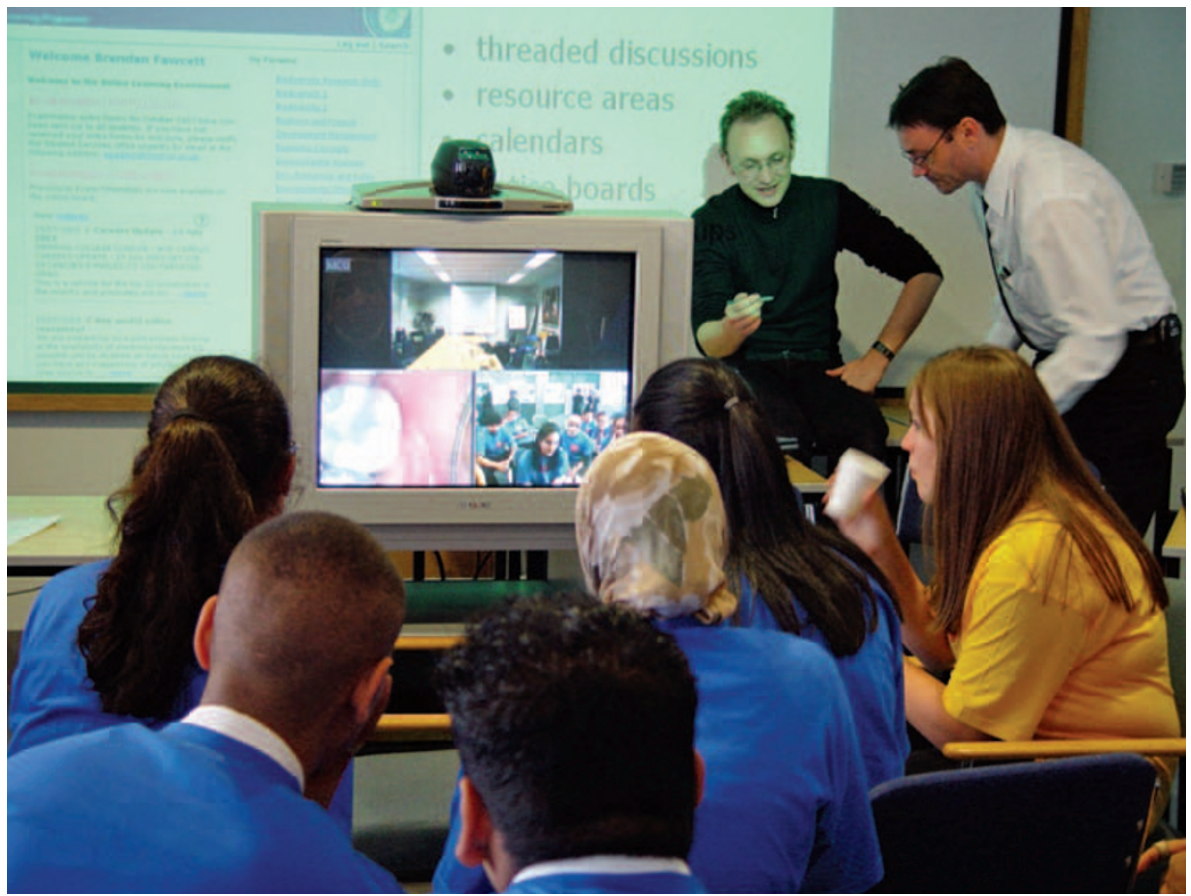

Fig. 2 Videoconferencing system with bridged link between three sites

\section{VIRTUAL LEARNING ENVIRONMENTS}

'Online learning is not the next big thing; it is the now big thing.'

Donna J. Abernathy, Editor of Training and Development, 1999.

Technological developments (eg www, digital satellite technology and innovative applications of virtual reality) are having dramatic effects on learning environments at all levels. Learning can be mediated through the web using lecturing and telling ('sage on the stage') to facilitating and guiding ('guide on the side') has emerged. ${ }^{18}$

To optimise the use of the Internet for education, the incorporation of active learning strategies into teaching must include more than just downloading material from the course. The value of well-delivered lectures is known despite the low level of participation from the audience. ${ }^{19}$ Web-based learning tools can challenge students with a task, provide them with resources that support higher or critical level thinking through active learning and have them work together collaboratively. ${ }^{20}$

Ten years ago, children were introduced to the computer by playing games; today they are introduced through the worldwide web, and are familiar with information at their fingertips. Programs enhanced by web-based tools have been shown to increase student performance by making them more confident and thus allowing them to deal with information in ways that make the material more relevant to their lives. ${ }^{21}$ There are a number of ways that these courses can transform teaching, learning, and the curriculum:

- Web-enhanced training programs can more actively engage students in the construction of their own knowledge by imbuing them with a learning responsibility. They have also moved learning from an individual, isolated act towards a collaborative activity where ideas are considered in a much wider context ${ }^{22}$

- Web tools, and ICT in general, remove the barriers between the classroom and the real world. Technology can expand a student's ability to express, understand and use ideas by referencing a worldwide resource, rather than a limited local resource ${ }^{22}$

- Web-based learning can empower students to use other ICT tools such as email, newsgroups etc, that facilitate the learning process

- Web-based tools and ICT can increase the quantity and quality of information shared between teachers and students. ${ }^{3,23}$

Methods of assuring quality must be incorporated into the programme. 
Teachers will need to embrace the features of an online pedagogy so that they can best use their traditional teaching resources, often to larger and geographically separated audiences. However, there are limits. The ideal numbers are thought to be between 20 and 35 students to tutor where an emailed response to textbook materials constitutes the learning environment. ${ }^{24}$

The integration of web-based learning tools into teaching should commence with simple exercises before using all the web-based learning components. Simple rules can keep order within the course. For instance, periods of time are defined when rapid response is guaranteed or when teachers will be in chat rooms. ${ }^{25}$ Principles of good online design will be discussed in a later article in this series.

A new generation of web applications is becoming available that combines data from more than one source into a single integrated tool (Mashups, www. mashups.com). Teaching and learning tools can also be linked together through a systems-based infrastructure - a sort of electronic super glue - called a service orientated architecture (SOA). This is forming the basis of VLE 2.0. ${ }^{26}$

\section{VIDEOCONFERENCING AND WEBCASTING}

\section{Videoconferencing}

Videoconferencing allows video, audio and data to be transmitted and received between two or more sites at the same time. Computer programs can also be shared. Videoconferencing can take a number of forms, which include:

- One-to-one meetings (or "point-topoint' communication), which use full two-way video and audio

- One-to-many involving full audio and video broadcast from a main site, with other, receiving sites having only an audio transmitting capability (eg a lecture from a main site, with remote audio allowing the distant students to ask questions)

- Many-to-many (known as 'multipoint' communication) which provides audio and video between two or more sites. Most multi-point systems allow only one site in the conference to be seen at a time, with switching between sites either controlled manually or voice activated.

To allow any of these forms of videoconferencing to take place, different types of equipment or 'hardware' is required. The most common types of videoconferencing equipment include:

- Desktop videoconferencing (a small camera is located on top of the PC with the video/audio input from the remote location displayed in a small window onscreen)

- Meeting room systems (a dedicated room is equipped for videoconferencing with cameras, microphones, display monitors (Fig. 2). Other equipment may also be deployed such as document viewers.

\section{Delivery}

Currently the delivery of videoconferencing is available through three 'carriers', ISDN, computer Internet protocol (IP)-based systems and satellite systems.

ISDN provides fast, high capacity digital transmission of voice, data, images and full-motion video and can be usually offered at 'basic rate' and 'primary rate'. Basic rate provides two lines at 64 Kbps each. This represents the number of data channels and one control channel allowing reasonable quality videoconferencing. Primary rate provides 30 x 64 Kbps data channels and one control channel and allows excellent quality video and audio. (It should be noted that primary rate services in nonEuropean countries such as the USA and Japan usually use 23 data channels and one control channel, which provides a lower bandwidth).

Videoconferencing systems increasingly incorporate the IP (Internet protocol) rather than just ISDN. Each computer is given a unique IP address (a special number). This allows one computer to link directly with another provided the addresses are known and permission granted. Many institutional networks have a pre-existing IP infrastructure, and some of the compression software required for efficient data transmission is available free. With improvements in IP transmission in terms of reliability and security it is possible that it will replace ISDN as the preferred delivery protocol for videoconferencing, thereby eliminating the disadvantage of the latter's call charges.

Satellite broadcast or satellite transmissions offer large transmission bandwidths and are normally used for one-to-many conferences. Although expensive, cost is not affected by distance and therefore it may be used where very large distances or many sites are involved.

\section{Using videoconferencing}

The basic hardware components for videoconferencing include CODEC, cameras, microphones, speakers (or headphones), video capture cards (video capture refers to storing video images in a computer) and network cards or ISDN adaptors (to connect the computer to the ISDN line). Most of these components are now packaged into a set-top box that connects to a standard TV screen, to which High Definition TV technology is increasingly being applied.

A wide range of software is available and its effectiveness depends on the kind of tasks it is expected to perform. 'Multipoint' (a method where data is only sent once but received by every participant) is one solution allowing many-tomany conferences over networks.

Videoconferencing can be used for a variety of purposes including personal communication, normally using desktop systems such as Skype ${ }^{\mathrm{TM}}$ (www.skype. com) or dedicated studios, collaborative work using shared applications, presentations and education involving one-to-many connections with student sites. However, it is most useful when there is a clear communication need as it can reduce travel costs, provide obvious enhancements over standard telephone conversations and provide a better environment for quality remote teaching. Projects at a postgraduate and undergraduate level in dentistry have clearly illustrated these points. The pilot regional online videoconferencing in dentistry (PROVIDENT) project linked four London teaching hospitals with eight postgraduate centres in the Thames regions. ${ }^{27,28}$ Dental undergraduate education by teleconferencing (DUET) linked a remote practice in the Isle of Wight with 


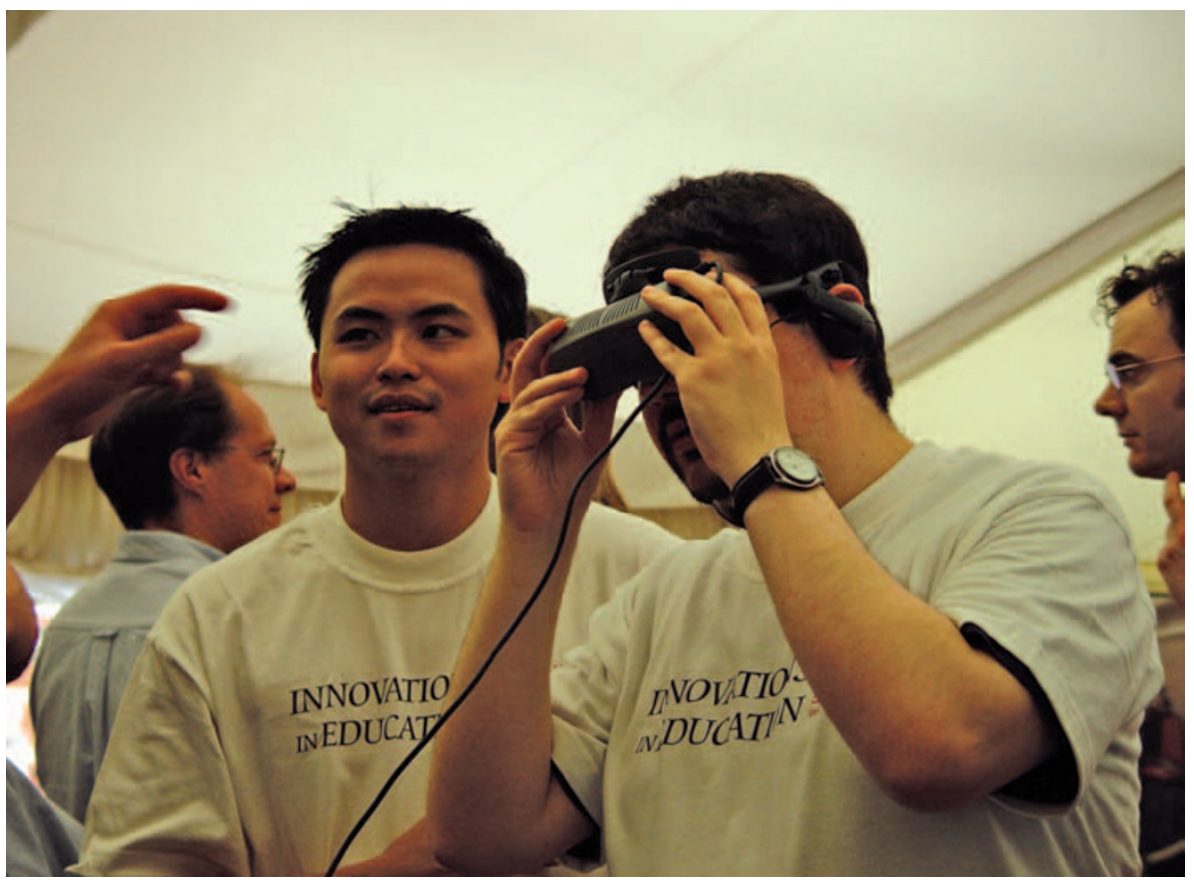

Fig. 3 Virtual reality headgear in use

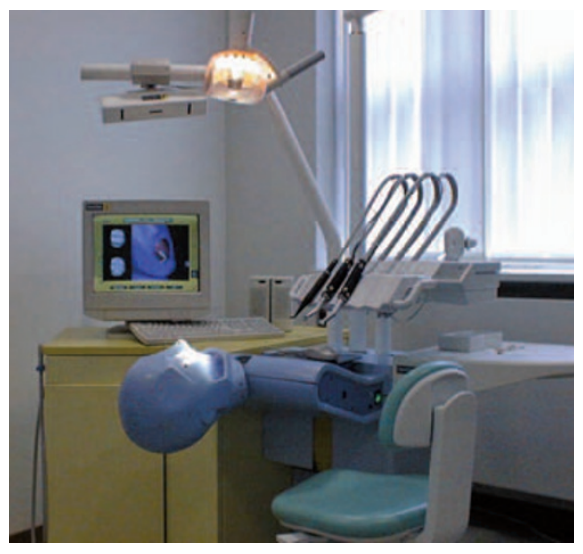

Fig. 4 DentSim simulator

students at a London teaching hospital 150 kilometres away. ${ }^{29}$ The Dublin Dental School has been using its videoconferencing facilities for one-to-one links but is expanding its capabilities throughout Ireland as part of its continuing dental education programme.

Videophones have provided a simple audiovisual communication alternative when the bandwidth has been restricted. Using satellite links, videophones have been used most recently for televised reports from war zones.

\section{Webcasting}

Webcasting, also referred to as 'netcasting' or 'Internet broadcasting', is the transmission of live or pre-recorded audio or video to computers that are connected to the Internet. Similar to television, radio or videoconferencing, webcasting provides 'one-to-many' communications allowing people to take part in an event or a presentation remotely. The advantage of webcasting over television or radio broadcasting is the availability of information globally, 24 hours a day, seven days a week. One of the most famous webcasts, which drew 9 million logons, was for a concert by singer Madonna in London in November 2002. ${ }^{30}$ This webcast was delivered by Microsoft to demonstrate the excellent video and audio quality webcasting can provide, although it also demonstrated the infancy of the technology as many 'virtual fans' were left disappointed as the huge demand on the webcast site led to numerous complaints of bad quality video and audio.

The technology that allows webcasting to take place is known as 'streaming media technology'. Streaming media technologies transmit audio and video from a centralised source, known as a 'server', to a client on a computer, known as a 'media player'. When the user requests a streaming media file, for example a prerecorded video/audio track or live transmission, the server sends a stream of data 'packets' (small groups of digital data) to the media player where it plays as it is received. It means that there is no need to download the video file which is likely to be enormous in size. The hardware and software requirements to allow a user to satisfactorily access a webcast are a PC complete with a sound card, speakers, connection to the Internet (minimum recommended connection speed is $28.8 \mathrm{Kbps}$ ) and media player software. Two popular streaming media players are the 'Real Player' by Real Networks (http://www. real.com) and the 'Windows Media Player' by Microsoft (http://www.windowsmedia. com), both of which can be can be downloaded free from the Internet.

Webcasting can be used for distance learning and education in a similar fashion to videoconferencing with the added benefit of being time-independent. Webcasting can also be used for press conferences and media events, live seminars and global company events and for delivering CPD. ${ }^{31}$

\section{Podcasting and RSS (real simple syndication) feeds}

A podcast is a media file that is distributed over the Internet for playback on portable media players (such as MP3 [moving picture experts group audio layer 3] or iPod ${ }^{\mathrm{TM}}$ players) and PCs. RSS is a family of web feed formats that allows users to subscribe to podcasts and publishes the latest digital content. ${ }^{32}$

\section{USE OF INTERACTIVE TELEVISION LINKS}

Interactive television links (iTV) is a format in which domestic television technology is blended with the interactive abilities usually associated with the Internet, DVD or other ICT tools. This technology is facilitated through a 'back channel' and/or an advanced set-top terminal allowing the provision of televisual interactive content for users and viewers. A set-top terminal or box is a device that serves as a gateway between the television and a telephone or cable connection. In interactive television networks, the set-top box receives digital signals and either converts them into analogue signals for display on an analogue television or a digital monitor or High Definition device. In addition, the set-top box receives user input, usually via infrared remote control, which is transmitted back to the network. Set-top boxes, which are almost as powerful as computers complete with software and hardware upgrade-ability, can combine 
many functions including television receiver, modem, Internet browser, electronic programme guide (EPG) and DVD player. iTV has many differing delivery systems, technical standards, uses and content, all of which have a common factor in that television nowadays provides entertainment, education and information to billions of people around the world and also contributes to their leisure and social lives.

Education can also be provided via television links to live or pre-recorded events. These links can be either via extra-terrestrial communication satellites or via cable links. The MedLive/International Online Academy project provided an example of the use of communication satellites to deliver continuing education to dentists in their own homes. ${ }^{33}$ Within MedLive, a range of both live and prerecorded programmes were transmitted from a central studio and received via settop boxes. This method offers the advantages of broadband technology to deliver high quality still and moving images and excellent quality sound. However, it has the disadvantages that communication satellite time is expensive and at present there is little potential for real-time direct interaction between the receiver and the broadcaster.

\section{VIRTUAL REALITY (VR) AND SIMULATION}

The ability to create 'virtual' or 'alternative' reality enables simulation of

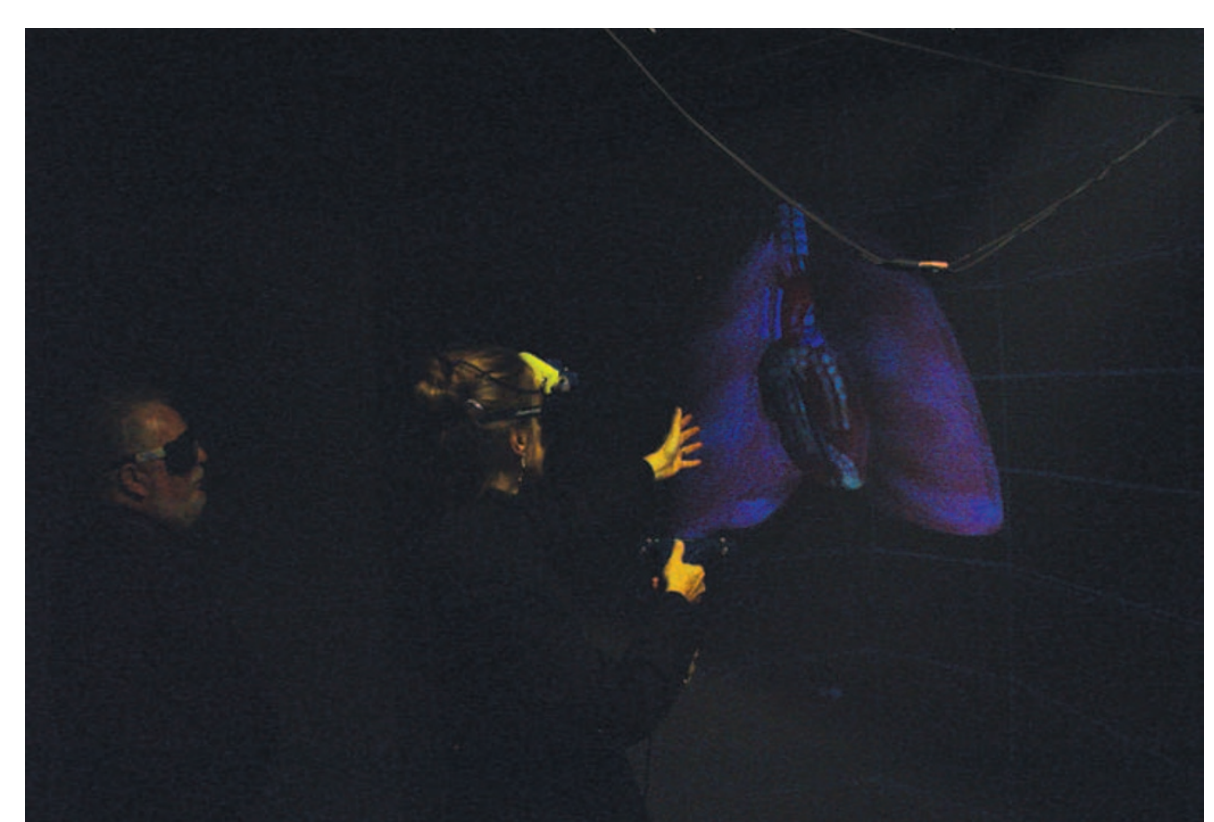

Fig. 5 Inside a CAVE (computer assisted virtual environment)

practical procedures in three dimensions. Although surgical simulation of operations using gloves with mini sensors, VR glasses and a remote robotic operator have been described, the main use of VR is still in games (Fig. 3). VR spectacles have been used as a distraction for patients undergoing dental treatment, but patients can become disorientated for some time after using the goggles and so should not operate machinery or drive a car for at least an hour following their use. ${ }^{34}$ Immersion chambers such as the CAVE (computer assisted virtual environment) can allow the user to walk through 3D images such as the heart and lungs, and manipulate them..$^{35}$

In the field of simulation, sophisticated PC-based devices have been created using multiple infrared (IR) laser emitting devices (LEDs) and IR sensors to register the relative position of the drill, phantom head and teeth accurately. DentSim is such a system which can precisely reproduce cavity and crown preparations giving real-time feedback in 3D to the operator (http://www. denx.com) (Fig. 4). These simulators are particularly valuable in pre-clinical and postgraduate training, in retraining and in the assessment of students. There is no doubt that future VR devices will provide accurate representations of many other procedures and will be valuable training simulators. However, the human element must never be dismissed, as the interaction with both a real tutor

BRITISH DENTAL JOURNAL VOLUME 204 NO. 1 JAN 122008 and patient add an important dimension to training.

Computer programmes (software) can also produce VR effects. Two- and threedimensional objects can be incorporated into the web that can be controlled by the viewer (Fig. 5). ${ }^{36}$

\section{CONCLUSIONS}

The use of the ICT is likely to continue to expand at an exponential rate of development in the future. The advent of computers will be as important as that of printing in facilitating the transfer of information to and between global populations. Computers and workstations are being manufactured with many of the required components as standard features, for example DVD drives and advanced video adaptors. Local and wide area networks will also evolve to cope with the increased data demands of these tools, now called Web 2.0. ${ }^{37}$ Time and travel savings and the already comprehensive literature on the use of ICT in education are compelling arguments to encourage its uptake in this field.

All educators should be encouraged to investigate the available ICT technologies and tools in the context of education - as Mason states, 'A good teacher is a good teacher in any medium'. ${ }^{38}$ Students can be provided with an enriched and diverse active learning environment which is student-centred and collaborative. It must be remembered that traditional methods occasionally break down too, eg slide projector bulbs break and the chalk goes missing. In the final analysis, dedication, application and patience are needed to ensure long-term gain as the challenge continues in education to prioritise the appropriate development and investment in ICT. However, the educational needs must drive the development of the appropriate technology, not the tools themselves looking for a purpose.

1. Laurillard D. Moving towards a unified e-learning strategy - an update. London: Department for Education and Skills, 2004.

2. Eisenstadt M, Vincent $\mathrm{T}$ (eds). The knowledge web learning and collaborating on the web. London: Kogan Page, 2000.

3. Bates A W. Technology, open learning and distance education. London: Routledge, 1997.

4. Mason R. Globalising education: trends and applications. London: Routledge, 1998.

5. Bates A W. Managing technological change: strategies for academic leaders. San Francisco: Jossey Bass, 1999.

6. Opportunities and barriers to the use of broadband 
in education. 2003. Broadband Stakeholder Group, 2003. http://www.broadbanduk.org/component/option,com_docman/task,doc_view/gid,54/ Itemid,9/. Accessed 24 February 2007.

7. Organisation for Economic Co-operation and Development. OECD broadband statistics December 2005. OECD, 2005.

8. Internet Usage Stats. Usage and population statistics January 2007. http://www.internetworldstats. com/top20.htm. Accessed 24 February 2007.

9. Levitt M. Email usage forecast and analysis, 2000-2005. IDC Report W23011. International Data Corporate, 2000.

10. JISC. In their own words. Exploring the learner's perspective on e-learning. Bristol: JISC, 2007. http://www.jisc.ac.uk/publications/publications/ intheirownwords.aspx. Accessed 4 November 2007.

11. Mason R, Rennie F. Elearning: the key facts. London: Routledge, 2006.

12. Reynolds P A. e-Learning - what is really needed? A commentary from Krakow. UK Health Informatics Today. Newsletter of the UK Informatics Society 2007; 52: 4-6.

13. Reynolds PA. Multimedia: the next generation's education. Proceedings of the University Teachers in Oral and Maxillofacial Surgery Meeting, Sheffield, 1997.

14. Towell P. Discussion of outline research proposal to create development, design and production methods for digital media founded on semiotic theory. London Knowledge Lab (Seminar). 1 November 2006. http://www.|kl.ac.uk/cms/ index.php?option $=$ com_events\&task=view_detai I \& tagid $=62$ \&tyear $=2006$ \&tmonth $=11$ \&tday $=01$ \& $\mid$ te mid=27. Accessed 24 February 2007.

15. Eaton K A. CAL for dentists - where have we been? Where are we going? 1999. http://www.eastman. ucl.ac.uk/cal/bscd99/04_eaton/index.html

16. Benedetto S. Highlights from Syllabus Magazine: DVD video: a primer for educators. Syllabus Magazine $2000 ; 14(1)$.

17. Harasim $L$, Hiltz $R$, Teles $L$, Turoff M. Learning networks: a field guide to teaching and learning online. Cambridge, Mass.: MIT Press, 1995.

18. Rowntree D. Developing a distance learning course. Open University, 2000. http://www-iet.open. ac.uk/pp/D.G.F.Rowntree/DL_course_develpt.html. Accessed 24 February 2007.

19. Dodge B. Active learning on the web. San Diego: San Diego State University, 2001. http://edweb.sdsu. edu/people/bdodge/Active/ActiveLearning.html.

20. Ninck A, Rollinghoff A. Scenarios for web-based collaborative learning. Proceedings 7 th International Conference on Computers in Education, Copenhagen Jul 29-Aug 32001.

21. Follansbee $S$, Hughes R, Pisha B, Stahl S. The role of online communications in schools: a national study 1996. Centre for Applied Special Technology, 1997.
22. Kosmer R B, Johnson J. The technological revolution comes to the classroom. Change 1991; Jan/Feb: $10-20$

23. Calder J. Programme evaluation and quality. London: Kogan Page, 1997.

24. Knox A B. Evaluating adult and continuing education. Columbus: ERIC Clearinghouse on Adult, Career and Vocational Education, 1998. Information Series No. 375.

25. Reynolds P A. Learning on-line: the virtual classroom of the 21st Century. Keynote address, Sixth International Symposium of Stomatology, Timisoara, Romania, 2001.

26. Weller M. VLE 2.0 and future directions in learning environments. In Philip R, Voerman A, Dalziel J (eds) Proceedings of the first international LAMS conference 2006: designing the future of learning. pp 99-106. Sydney: LAMS Foundation, 2006. http://lamsfoundation.org/lams2006/papers.htm. Accessed 4 November 2007.

27. Eaton K A, Francis C A, Odell E W, Reynolds PA, Mason R D. Participating dentists assessment of the pilot regional online videoconferencing in dentistry (PROVIDENT) project. Br Dent J 2001; 191: 330-334.

28. Odell E W, Francis C A, Eaton K A, Reynolds P A Mason R D. A study of videoconferencing for postgraduate continuing education in dentistry in the UK - the teachers' view. Eur J Dent Educ 2001; 5: $113-110$.

29. Reynolds P A. Dental undergraduate education by teleconferencing (DUET): experiences over 4 years. (Videoconferenced paper). Proceedings of the Sixth International Conference on Medical Aspects of Telemedicine, Uppsala, Sweden, June 18-21, 2001.

30. Mariano G. Madonna webcast shows Internet is not TV. CNET News, 28 November 2000. http:// news.cnet.com/news/0-1005-200-3890055.htm

31. Richardson W. Blogs, wikis and podcasts and other powerful tools for classrooms. London: Sage Publications Ltd, 2006.

32. Reynolds P A, Mason R D. Online videomedia for the continuing professional development in dentistry. Comput Educ 2002; 39: 65-98.

33. Fuchs B. Prasenzakademie und Online Academy Das DGZMK/APW- Modell zur Strukturierten curricularen Fortbildung. Quintessenz 2000; 51(10). [In German].

34. McCloy R, Stone R. Science, medicine and the future: virtual reality in surgery. BMJ 2001; 323: 912-915.

35. Reading Visualisation Centre. University of Reading. http://www.rvc.reading.ac.uk/. Accessed 24 February 2007.

36. Mohler J L. Improving spacial ability with virtual reality: a review of research and applications. WebNet J 2001; 3(1): 28-35.

37. O'Reilly T. What is web 2.0? Design patterns and business models for the next generation of software. 30 September 2005. http://www.oreillynet. com/pub/a/oreilly/tim/news/2005/09/30/whatis-web-20.html. Accessed 24 February 2007.

38. Mason R. Moderating educational computer conferencing. DEOSNEWS 1991; vol. 1 no. 19 http://www.emoderators.com/papers/mason.html

\section{GLOSSARY OF TERMS}

3G - Third generation networks capable of transmitting video ADSL - Asynchronous digital service lines

BD - Blu-ray definition

BSG - Broadband Stakeholder Group

CAVE - Computer assisted virtual environment

CD-ROM - Compact disc-read only memory

DVD - Digital versatile/video disc

EU - European Union

HD - High definition video

IP - Internet protocol - postal system addressing for individual computers ISDN - Integrated services digital network

JANET - Joint academic network - superfast academic network MP3 - Moving picture experts group audio layer 3

OECD - Organisation of Economic Cooperation and Development PAL - Phased alternating line RSS - Real simple syndication SDSL - Synchronous digital service lines

SHDSL - Symmetric services, offering higher speeds in both directions SOA - Service orientated architecture USB - Universal serial bus VR - Virtual reality 